\title{
Changing blow-up time in nonlinear Schrödinger equations
}

\author{
Rémi Carles
}

\begin{abstract}
Solutions to nonlinear Schrödinger equations may blow up in finite time. We study the influence of the introduction of a potential on this phenomenon. For a linear potential (Stark effect), the blow-up time remains unchanged, but the location of the collapse is altered. The main part of our study concerns isotropic quadratic potentials. We show that the usual (confining) harmonic potential may anticipate the blow-up time, and always does when the power of the nonlinearity is $L^{2}$-critical. On the other hand, introducing a "repulsive" harmonic potential prevents finite time blow-up, provided that this potential is sufficiently "strong". For the $L^{2}$-critical nonlinearity, this mechanism is explicit: according to the strength of the potential, blow-up is first delayed, then prevented.
\end{abstract}

\section{Introduction}

Consider the nonlinear Schrödinger equation with power-like nonlinearity in $\mathbb{R}^{n}$,

$$
\left\{\begin{aligned}
i \partial_{t} v+\frac{1}{2} \Delta v & =\lambda|v|^{2 \sigma} v, \\
v_{\mid t=0} & =u_{0} .
\end{aligned}\right.
$$

Many results are available on the local and global existence issues for this initial value problem (see e.g. [10]). If $u_{0} \in H^{1}\left(\mathbb{R}^{n}\right), \lambda<0, \sigma \geq 2 / n$ and $\sigma<2 /(n-2)$ when $n \geq 3$, (1.1) has a unique solution, which is defined locally in time, $v \in$ $C\left([0, T] ; H^{1}\left(\mathbb{R}^{n}\right)\right)$. It may not be global. Define

$$
\Sigma:=\left\{f \in H^{1}\left(\mathbb{R}^{n}\right) ;|x| f \in L^{2}\left(\mathbb{R}^{n}\right)\right\} .
$$

If we assume moreover $u_{0} \in \Sigma$, and

$$
E\left(u_{0}\right):=\frac{1}{2}\left\|\nabla u_{0}\right\|_{L^{2}}^{2}+\frac{\lambda}{\sigma+1}\left\|u_{0}\right\|_{L^{2 \sigma+2}}^{2 \sigma+2}<0
$$

MSC 2000: 35Q55, 35B33.

Keywords : finite time blow-up, nonlinear Schrödinger equation, Stark effect, harmonic potential. 
then $v$ blows up in finite time, that is there exists $T>0$ such that

$$
\lim _{t \rightarrow T}\left\|\nabla_{x} v(t)\right\|_{L^{2}}=+\infty
$$

This is proven by the general approach of Zakharov-Glassey ([16], [10]). In some particular cases, the blow-up phenomenon is well understood; see for instance [18], [1], [21], [19], [20].

The introduction of a stochastic white noise in (1.1) may amplify or prevent blow-up formation (see [14, 13], and references therein); we consider a deterministic framework.

It is shown in [12] that if the initial datum $u_{0}$, such that (1.2) holds, is replaced by $u_{0}(x) e^{-i b|x|^{2}}$ with $b>0$ sufficiently large, then the blow-up time is anticipated (and is $O\left(b^{-1}\right)$ ). On the other hand, if $u_{0}$ is replaced by $u_{0}(x) e^{i b|x|^{2}}$ with $b>0$ sufficiently large, then no blow-up occurs. We want to obtain similar results without modifying the initial datum, but by introducing a potential,

$$
\left\{\begin{aligned}
i \partial_{t} u+\frac{1}{2} \Delta u & =V(x) u+\lambda|u|^{2 \sigma} u, \\
u_{\mid t=0} & =u_{0} .
\end{aligned}\right.
$$

Our results are suggested by the semi-classical régime for linear Schrödinger equation with potential. Consider the initial value problem

$$
\left\{\begin{aligned}
i \varepsilon \partial_{t} u^{\varepsilon}+\frac{1}{2} \varepsilon^{2} \Delta u^{\varepsilon} & =V(x) u^{\varepsilon}, \quad(t, x) \in \mathbb{R} \times \mathbb{R}^{n}, \\
u_{\mid t=0}^{\varepsilon} & =u_{0}^{\varepsilon},
\end{aligned}\right.
$$

where $\left.\left.V \in C^{\infty}\left(\mathbb{R}^{n}, \mathbb{R}\right), \varepsilon \in\right] 0,1\right]$. In the semi-classical limit $\varepsilon \rightarrow 0$, the energy of the solution $u^{\varepsilon}$ is carried by bicharacteristics, which are the integral curves associated to the classical Hamiltonian

$$
p(t, x, \tau, \xi)=\tau+\frac{|\xi|^{2}}{2}+V(x) .
$$

If the energy tends to concentrate in this case, one can expect that for (1.3) with a focusing nonlinearity $(\lambda<0)$, blow-up in finite time, which corresponds to the concentration of the mass, may occur. Bicharacteristic curves solve

$$
\dot{t}=1 ; \quad \dot{x}=\xi ; \quad \dot{\tau}=0 ; \quad \dot{\xi}=-\nabla V(x) \text {. }
$$

Rays of geometric optics, which are the projection of bicharacteristic curves on $(t, x)$-space, are of the form $x=x(t)$, with

$$
\ddot{x}+\nabla V(x)=0 ; x(0)=x_{0}, \dot{x}(0)=\xi_{0} .
$$

If the initial datum is of the form $u_{0}^{\varepsilon}(x)=f(x) e^{i \varphi(x) / \varepsilon}$, then $\xi_{0}=\nabla \varphi\left(x_{0}\right)$. We give four examples which correspond to cases where (1.5) can easily be solved. 

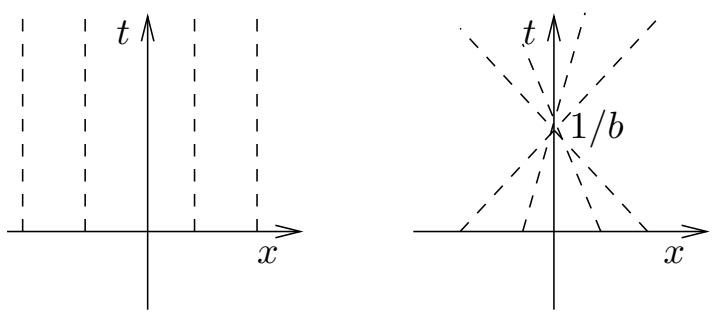

$\varphi \equiv 0$

$$
\varphi(x)=-b|x|^{2} / 2
$$

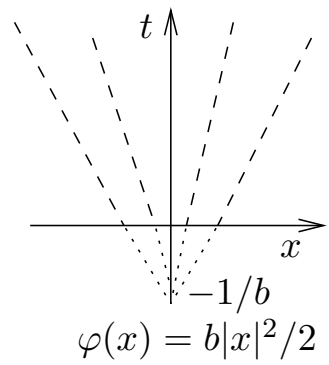

Figure 1: Geometry of rays: case $V \equiv 0$.

Example 1. Suppose $V \equiv 0$. Then the solutions of (1.5) are

$$
x(t)=x_{0}+t \nabla \varphi\left(x_{0}\right) .
$$

If $\varphi \equiv 0$, rays are parallel. More interesting is the case of quadratic oscillations (see also [8]). If $\varphi(x)=-b|x|^{2} / 2$ with $b>0$, then rays are given by $x(t)=x_{0}(1-b t)$, and meet at the origin at time $t=1 / b$ (see Figure 1 ). There is focusing, which suggests that in a nonlinear setting, such oscillations may cause wave collapse. If $\varphi(x)=b|x|^{2} / 2$ with $b>0$, then rays are given by $x(t)=x_{0}(1+b t)$, and met at the origin at time $t=-1 / b$ (in the past). In particular, they are spread out for positive times, which suggests that in a nonlinear setting, such oscillations may prevent wave collapse. The intuition described on the last two cases is confirmed by the results of Cazenave and Weissler [12].

Example 2. If $V(x)=E \cdot x$ is a Stark potential $\left(E \in \mathbb{R}^{n}\right.$ is constant), then

$$
x(t)=x_{0}+t \nabla \varphi\left(x_{0}\right)-\frac{1}{2} E t^{2} .
$$

If $\varphi \equiv 0$, rays do not meet (see Figure 2). If $\varphi(x)=-b|x|^{2} / 2$ with $b>0$, then rays

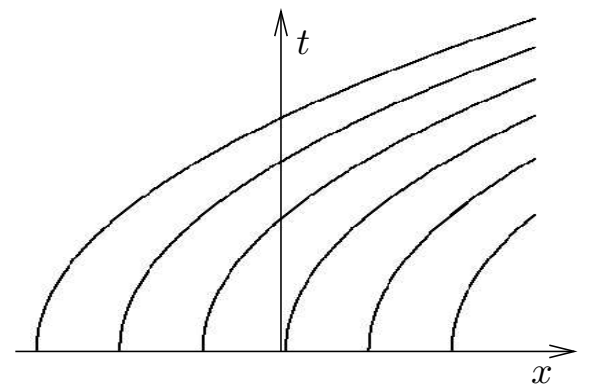

$\varphi \equiv 0$

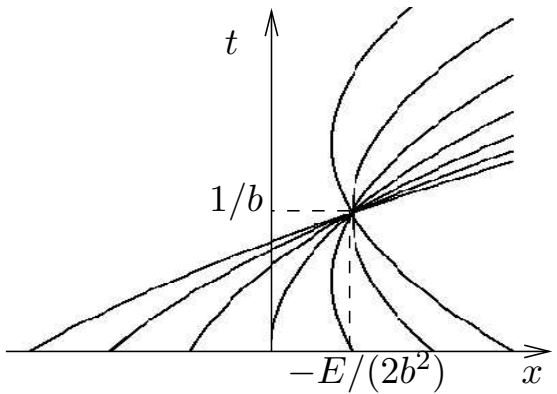

$\varphi(x)=-b|x|^{2} / 2$

Figure 2: Geometry of rays: case $V(x)=E \cdot x$.

meet at the point $-E /\left(2 b^{2}\right)$ at time $t=1 / b$ (see Figure 2). Compare this with the second case of the first example. This suggests that in a nonlinear setting, a linear potential does not affect the blow-up time, but the location when this phenomenon occurs. 
Example 3. Suppose $V(x)=\omega^{2}|x|^{2} / 2$, with $\omega>0$. In the case $\varphi \equiv 0$, rays are given by $x(t)=x_{0} \cos (\omega t)$, and meet at the origin at time $t=\pi /(2 \omega)$ (see Figure 3). The first example suggests that blow-up may happen more easily than when $V \equiv 0$. This phenomenon is reinforced by the case $\varphi(x)=-\omega \tan \left(\omega t_{0}\right)|x|^{2} / 2,\left|t_{0}\right|<\pi /(2 \omega)$, where

$$
x(t)=\frac{x_{0}}{\cos \left(\omega t_{0}\right)} \cos \omega\left(t+t_{0}\right) .
$$

Rays meet at the origin at time $t=\pi /(2 \omega)-t_{0}$. If $t_{0}>0$, focusing is anticipated, while if $t_{0}<0$, it is delayed (but in no case prevented).

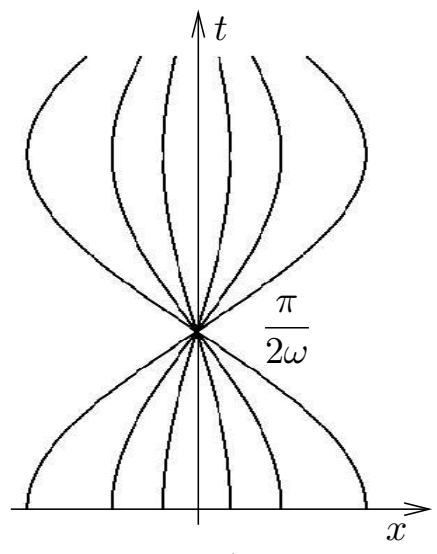

$$
V(x)=\frac{1}{2} \omega^{2}|x|^{2}
$$

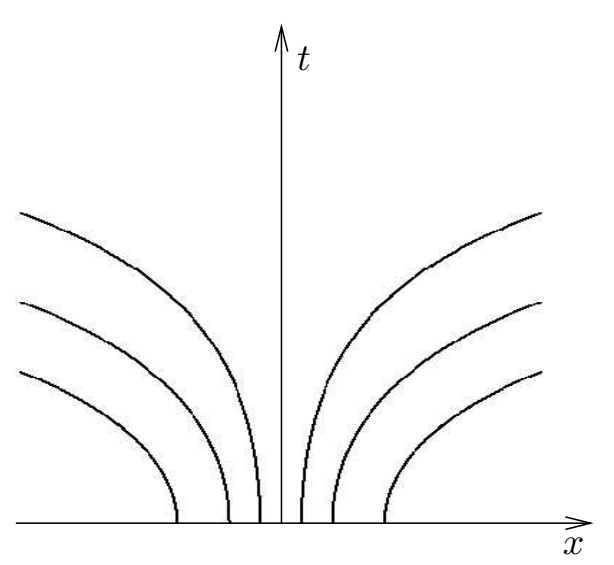

$V(x)=-\frac{1}{2} \omega^{2}|x|^{2}$

Figure 3: Geometry of rays, with $\varphi \equiv 0$.

Example 4. Suppose $V(x)=-\omega^{2}|x|^{2} / 2$, with $\omega>0$. In the case $\varphi \equiv 0$, rays are given by $x(t)=x_{0} \cosh (\omega t)$, and are strongly dispersed for positive times (see Figure 3). This geometry is to be compared with the third case of the first example; rays are scattered, but go to infinity exponentially fast, instead of algebraically. This is a hint that such potentials may prevent blow-up.

We prove that in the last three examples, our heuristic approach agrees with rigorous results.

Proposition 1.1 (Stark potential, [9]). Let $E \in \mathbb{R}^{n}$, and $V(x)=E \cdot x$. Then the solutions $u$ and $v$ to (1.3) and (1.1) are related through the formula

$$
u(t, x)=v\left(t, x+\frac{t^{2}}{2} E\right) e^{-i\left(t E \cdot x+\frac{t^{3}}{6}|E|^{2}\right)} .
$$

In particular, if $v$ blows up at time $t=T$, then so does $u$, but the set where this phenomenon occurs is shifted by a factor $-T^{2} E / 2$.

Remark. In the linear case $\lambda=0$, the above formula is the well-known Avron-Herbst formula (see e.g. [11], Chapter 7). It yields interesting results in the nonlinear setting, as shown by the above proposition. 
We now turn to the case of isotropic quadratic potentials. Let $\omega>0$. Introduce the solutions $u_{+}^{\omega}$ and $u_{-}^{\omega}$ to the initial value problems

$$
\left\{\begin{aligned}
i \partial_{t} u_{ \pm}^{\omega}+\frac{1}{2} \Delta u_{ \pm}^{\omega} & = \pm \omega^{2} \frac{|x|^{2}}{2} u_{ \pm}^{\omega}+\lambda\left|u_{ \pm}^{\omega}\right|^{2 \sigma} u_{ \pm}^{\omega}, \quad(t, x) \in \mathbb{R} \times \mathbb{R}^{n} \\
u_{ \pm \mid t=0}^{\omega} & =u_{0} .
\end{aligned}\right.
$$

Remark. The plus case corresponds to the current model used to analyze BoseEinstein condensation. The harmonic potential is due to a magnetic trap, its confining properties are used to form a condensate of atoms. The nonlinear term corresponds to the interaction of particles. The real $\lambda$ may be positive or negative, according to the chemical element considered (see e.g. [3], [2], [23]). The power of the nonlinearity is $\sigma=2$ if $n=1, \sigma=1$ if $n=2$ or 3 (see e.g. [17]).

Theorem $1.2([4],[7])$. Let $u_{0} \in \Sigma, \lambda<0, \sigma \geq 2 / n$ and $\sigma<2 /(n-2)$ if $n \geq 3$. 1. Assume that (1.2) holds. Then $v$ blows up in finite time $T$, and $u_{+}^{\omega}$ blows up at time $T_{+}^{\omega} \leq \pi /(2 \omega)$. Let $\omega_{0}=\pi /(2 T)$; then for any $\omega \geq \omega_{0}$, $u_{+}^{\omega}$ blows up before time $T$.

2. If the initial datum $u_{0}$ satisfies

$$
\frac{1}{2}\left\|\nabla u_{0}\right\|_{L^{2}}^{2}+\frac{\lambda}{\sigma+1}\left\|u_{0}\right\|_{L^{2 \sigma+2}}^{2 \sigma+2}<-\frac{\omega^{2}}{2}\left\|x u_{0}\right\|_{L^{2}}^{2}
$$

then $u_{-}^{\omega}$ blows up in finite time, in the future or in the past.

3. If the initial datum $u_{0}$ satisfies

$$
\frac{1}{2}\left\|\nabla u_{0}\right\|_{L^{2}}^{2}+\frac{\lambda}{\sigma+1}\left\|u_{0}\right\|_{L^{2 \sigma+2}}^{2 \sigma+2}<-\frac{\omega^{2}}{2}\left\|x u_{0}\right\|_{L^{2}}^{2}-\omega\left|\operatorname{Im} \int \overline{u_{0}} x \cdot \nabla_{x} u_{0}\right|,
$$

then $u_{-}^{\omega}$ blows up in finite time, in the future and in the past.

4. There exists $\omega_{1}>0$ such that for any $\omega \geq \omega_{1}, u_{-}^{\omega}$ is globally defined, $u_{-}^{\omega} \in$ $C(\mathbb{R}, \Sigma)$.

In the particular $L^{2}$-critical case, we can give more precise results.

Theorem 1.3 ([5], [7]). Let $u_{0} \in \Sigma, \lambda<0, \sigma=2 / n$. Assume that the solution $v$ to (1.1) blows up at time $T>0$.

- For any $\omega>0, u_{+}^{\omega}$ blows up at time $\arctan (\omega T) / \omega<T$.

- If $\omega<1 / T$, then $u_{-}^{\omega}$ blows up at time $\arg \tanh (\omega T) / \omega>T$.

- If $\omega \geq 1 / T$, then $u_{-}^{\omega}$ is globally defined in the future, $u_{-}^{\omega} \in C\left(\mathbb{R}_{+}, \Sigma\right)$.

In Section 2, we prove the first point of Theorem 1.2, and we sketch the proof of the last point in Section 3. The second and third points can be proved by using the general approach of Zakharov-Glassey (see [7]). In Section 4, we show why Theorem 1.3 holds. 


\section{Confining harmonic potential}

Assume $u_{0} \in \Sigma, \sigma \geq 2 / n$ and $\sigma<2 /(n-2)$ if $n \geq 3$. Assume also $\lambda \in \mathbb{R}$. Only later will we restrict to the case $\lambda<0$.

Consider the solution $u_{+}^{\omega}$, which is known to exist in $\Sigma$, locally in time (see [10]). The following quantities are independent of time,

Mass: $M=\left\|u_{+}^{\omega}(t)\right\|_{L^{2}}$,

Energy: $E_{+}^{\omega}=\frac{1}{2}\left\|\nabla_{x} u_{+}^{\omega}(t)\right\|_{L^{2}}^{2}+\frac{\omega^{2}}{2}\left\|x u_{+}^{\omega}(t)\right\|_{L^{2}}^{2}+\frac{\lambda}{\sigma+1}\left\|u_{+}^{\omega}(t)\right\|_{L^{2 \sigma+2}}^{2 \sigma+2}$.

It is well known that if $E_{+}^{\omega}<0$, then $u_{+}^{\omega}$ blows up in finite up (see [10]). Notice that the assumption (1.2) is weaker.

The tools we use are suggested by geometric optics. The solution to (1.4) with $V(x)=\omega^{2}|x|^{2} / 2$ is given explicitly by Mehler's formula (see e.g. [15]), for $|t|<$ $\pi /(2 \omega)$

$$
u^{\varepsilon}(t, x)=e^{-i n \frac{\pi}{4} \operatorname{sgn} t}\left|\frac{\omega}{2 \pi \varepsilon \sin \omega t}\right|^{n / 2} \int_{\mathbb{R}^{n}} e^{\frac{i \omega}{\varepsilon \sin \omega t}\left(\frac{|x|^{2}+|y|^{2}}{2} \cos \omega t-x \cdot y\right)} u_{0}^{\varepsilon}(y) d y .
$$

If $u_{0}^{\varepsilon}=f$ is independent of $\varepsilon$, then stationary phase formula yields, for $0<t<\pi / 2 \omega$,

$$
u^{\varepsilon}(t, x) \underset{\varepsilon \rightarrow 0}{\sim} \frac{1}{(\cos \omega t)^{n / 2}} f\left(\frac{x}{\cos \omega t}\right) e^{-i \omega \frac{|x|^{2}}{2 \varepsilon} \tan \omega t} .
$$

With the study of a nonlinear problem in mind (especially in a semi-classical régime, see [6]), this suggests to introduce the operator

$$
\cos (\omega t) \times e^{-i \omega \frac{|x|^{2}}{2 \varepsilon} \tan \omega t} \nabla_{x}\left(\cdot e^{i \omega \frac{|x|^{2}}{2 \varepsilon} \tan \omega t}\right)=i \omega \frac{x}{\varepsilon} \sin \omega t+\cos \omega t \nabla_{x} .
$$

The above operator is well-known in the linear theory; this is an Heisenberg observable (see e.g. [22] p. 108). The factorization by which we retrieve this operator is of particular interest to treat nonlinear problems. Now set $\varepsilon=1$, and introduce the operators

$$
J_{+}^{\omega}(t):=-\omega x \sin \omega t+i \cos \omega t \nabla_{x} ; \quad H_{+}^{\omega}(t):=x \cos \omega t+i \frac{\sin \omega t}{\omega} \nabla_{x} .
$$

We can split the energy into two parts,

$$
\begin{aligned}
& E_{1}(t):=\frac{1}{2}\left\|J_{+}^{\omega}(t) u_{+}^{\omega}\right\|_{L^{2}}^{2}+\frac{\lambda}{\sigma+1} \cos ^{2} \omega t\left\|u_{+}^{\omega}(t)\right\|_{L^{2 \sigma+2}}^{2 \sigma+2}, \\
& E_{2}(t):=\frac{\omega^{2}}{2}\left\|H_{+}^{\omega}(t) u_{+}^{\omega}\right\|_{L^{2}}^{2}+\frac{\lambda}{\sigma+1} \sin ^{2} \omega t\left\|u_{+}^{\omega}(t)\right\|_{L^{2 \sigma+2}}^{2 \sigma+2} .
\end{aligned}
$$

We check that $E_{1}(t)+E_{2}(t) \equiv E_{+}^{\omega}$. It is proved in [4] that the evolution of $E_{1}$ is given by

$$
\frac{d E_{1}}{d t}=-\frac{d E_{2}}{d t}=\frac{\omega \lambda}{2 \sigma+2}(n \sigma-2) \sin 2 \omega t\|u(t)\|_{L^{2 \sigma+2}}^{2 \sigma+2} .
$$


The first point of Theorem 1.2 follows easily. Assume that $E\left(u_{0}\right) \leq 0$, which is even a weaker assumption than (1.2), and suppose that $u_{+}^{\omega}$ exists in $\Sigma$ up to time $\pi /(2 \omega)$. Then $E_{1}(0)=E\left(u_{0}\right) \leq 0$, and from (2.2), if $\sigma \geq 2 / n$ and $\lambda<0$,

$$
\frac{d E_{1}}{d t} \leq 0, \quad \forall t \in\left[0, \frac{\pi}{2 \omega}\right]
$$

This implies

$$
E_{1}\left(\frac{\pi}{2 \omega}\right) \leq 0
$$

But from the definition of $E_{1}$,

$$
E_{1}\left(\frac{\pi}{2 \omega}\right)=\frac{\omega^{2}}{2}\left\|x u_{+}^{\omega}\left(\frac{\pi}{2 \omega}\right)\right\|_{L^{2}}^{2},
$$

so this leads to a contradiction (unless $u_{+}^{\omega} \equiv 0$, which means that $u_{0} \equiv 0$ ). Therefore, $u_{+}^{\omega}$ does not remains in $\Sigma$ up to time $\pi /(2 \omega)$. It is easy to conclude that this is so because there exists $T \leq \pi /(2 \omega)$ such that

$$
\lim _{t \rightarrow T}\left\|\nabla_{x} u_{+}^{\omega}(t)\right\|_{L^{2}}=\infty .
$$

\section{Repulsive harmonic potential}

The most important step to study $u_{-}^{\omega}$ is the analysis of the Cauchy problem.

Formally, (1.7) with the plus sign is turned into (1.7) with the minus sign by replacing $\omega$ with $i \omega$ (or $-i \omega)$. Therefore, define

$$
J_{-}^{\omega}(t):=\omega x \sinh \omega t+i \cosh \omega t \nabla_{x} ; \quad H_{-}^{\omega}(t):=x \cosh \omega t+i \frac{\sinh \omega t}{\omega} \nabla_{x} .
$$

Introduce the evolution group associated to the linear equation $(\lambda=0)$,

$$
U_{-}^{\omega}(t):=\exp \left\{-i t / 2\left(-\Delta-\omega^{2}|x|^{2}\right)\right\} .
$$

- The first thing to notice is that $U_{-}^{\omega}(t)$ can be computed explicitly (essentially, trigonometric functions in (2.1) are turned into hyperbolic functions), and satisfies a dispersion estimate which is independent of $\omega>0$,

$$
\left\|U_{-}^{\omega}(t)\right\|_{L^{1} \rightarrow L^{\infty}} \leq\left|\frac{\omega}{2 \pi \sinh \omega t}\right|^{n / 2} \leq \frac{1}{|2 \pi t|^{n / 2}} .
$$

The group $U_{-}^{\omega}(t)$ thus yields the same Strichartz inequalities as in the case $\omega=0$, with constants independent of $\omega>0$.

- Duhamel's principle for $u_{-}^{\omega}$ is

$$
u_{-}^{\omega}(t)=U_{-}^{\omega}(t) u_{0}-i \lambda \int_{0}^{t} U_{-}^{\omega}(t-s)\left|u_{-}^{\omega}\right|^{2 \sigma} u_{-}^{\omega}(s) d s .
$$

The operators $J_{-}^{\omega}(t)$ and $H_{-}^{\omega}(t)$ satisfy the same properties as those which are used to study (1.1) in $\Sigma$ (which are $\nabla_{x}$ and $x+i t \nabla_{x}$ ), namely: 
- They commute with the linear part of the equation.

- They act on the nonlinear term like derivatives.

- They yield weighted Gagliardo-Nirenberg inequalities.

We make the last statement precise. Because $J_{-}^{\omega}(t)$ can be factorized in a similar fashion as $J_{+}^{\omega}(t)$, Gagliardo-Nirenberg inequalities imply

$$
\|f\|_{L^{r}} \leq \frac{C_{r}}{(\cosh \omega t)^{\delta(r)}}\|f\|_{L^{2}}^{1-\delta(r)}\left\|J_{-}^{\omega}(t) f\right\|_{L^{2}}^{\delta(r)}
$$

where

$$
\delta(r):=n\left(\frac{1}{2}-\frac{1}{r}\right), \text { and } 2 \leq r<\frac{2 n}{n-2} .
$$

Since $\cosh \omega t \geq 1$, the above estimate imply a Gagliardo-Nirenberg inequality involving only $\|f\|_{L^{2}}$ and $\left\|J_{-}^{\omega}(t) f\right\|_{L^{2}}$, with a weight independent of $\omega>0$.

- We infer that there exist $t_{0}>0$ independent of $\omega>0$, and a unique solution $u_{-}^{\omega}$ defined for $|t|<2 t_{0}$ with values in $\Sigma$, to (1.7) with the minus sign. Moreover, there exists $C_{0}$ depending only on $\lambda, n, \sigma$ and $\left\|u_{0}\right\|_{\Sigma}$ such that

$$
\sum_{A \in\left\{I d, J_{-}^{\omega}, H_{-}^{\omega}\right\}} \sup _{|t| \leq t_{0}}\left\|A(t) u_{-}^{\omega}\right\|_{L^{2}} \leq C_{0} .
$$

- Define

$$
E_{1}(t):=\frac{1}{2}\left\|J_{-}^{\omega}(t) u_{-}^{\omega}\right\|_{L^{2}}^{2}+\frac{\lambda}{\sigma+1} \cosh ^{2} \omega t\left\|u_{-}^{\omega}(t)\right\|_{L^{2 \sigma+2}}^{2 \sigma+2} .
$$

It solves, so long as $u_{-}^{\omega}$ is defined in $\Sigma$,

$$
\frac{d E_{1}}{d t}=\frac{\omega \lambda}{2 \sigma+2}(2-n \sigma) \sinh 2 \omega t\left\|u_{-}^{\omega}(t)\right\|_{L^{2 \sigma+2}}^{2 \sigma+2} .
$$

- This evolution law, its analog for $H_{-}^{\omega}(t) u_{-}^{\omega}$, along with the properties of $J_{-}^{\omega}(t)$ and $H_{-}^{\omega}(t)$, the algebraic relation

$$
\left\|J_{-}^{\omega}(t) u_{-}^{\omega}\right\|_{L^{2}}^{2}-\omega^{2}\left\|H_{-}^{\omega}(t) u_{-}^{\omega}\right\|_{L^{2}}^{2}=\left\|\nabla_{x} u_{-}^{\omega}(t)\right\|_{L^{2}}^{2}-\omega^{2}\left\|x u_{-}^{\omega}(t)\right\|_{L^{2}}^{2},
$$

and the conservations of mass and energy, imply the following crucial result.

Corollary 3.1. Let $\lambda \in \mathbb{R}, \sigma, \omega>0$, with $\sigma<2 /(n-2)$ if $n \geq 3$.

1. Let $u_{0} \in \Sigma$, and $u_{-}^{\omega}$ solve (1.7) on some time interval $I$ containing 0 . For any $I \ni t>0$, the following properties are equivalent:

- $\nabla_{x} u_{-}^{\omega} \in L^{\infty}\left([0, t] ; L^{2}\right)$.

- $u_{-}^{\omega} \in L^{\infty}([0, t] ; \Sigma)$.

- $J_{-}^{\omega}(s) u_{-}^{\omega}$ or $H_{-}^{\omega}(s) u_{-}^{\omega}$ is in $L^{\infty}\left([0, t] ; L^{2}\right)$.

- $J_{-}^{\omega}(s) u_{-}^{\omega}$ and $H_{-}^{\omega}(s) u_{-}^{\omega}$ are in $L^{\infty}\left([0, t] ; L^{2}\right)$. 
2. There exists a unique maximal solution, defined on $]-T_{*}, T^{*}\left[\right.$. If $T^{*}<\infty$, then $\left\|\nabla_{x} u_{-}^{\omega}(t)\right\|_{L^{2}} \rightarrow \infty$ as $t \uparrow T^{*}$.

Remark. Thanks to this result and to (3.2), we can prove that when $\lambda>0$, then $u_{-}^{\omega}$ is global, and a complete scattering theory is available (with no long range effect, see [7]). This result, if expected (the nonlinearity is repulsive), is not obvious from the usual conservations of mass and energy. The energy,

$$
E_{-}^{\omega}=\frac{1}{2}\left\|\nabla_{x} u_{+}^{\omega}(t)\right\|_{L^{2}}^{2}-\frac{\omega^{2}}{2}\left\|x u_{+}^{\omega}(t)\right\|_{L^{2}}^{2}+\frac{\lambda}{\sigma+1}\left\|u_{+}^{\omega}(t)\right\|_{L^{2 \sigma+2}}^{2 \sigma+2},
$$

is not the sum of positive terms, so we cannot conclude at this stage. On the other hand, Corollary 3.1 and (3.2) easily yield global existence.

- We can now prove the last point of Theorem 1.2. For $t \geq t_{0}$, integrate (3.2) between time $t_{0}$ and time $t$. Since $\lambda(2-n \sigma) \geq 0$,

$$
\begin{aligned}
E_{1}(t) & \leq E_{1}\left(t_{0}\right)+C \omega \int_{t_{0}}^{t} \sinh 2 \omega s\left\|u_{-}^{\omega}(s)\right\|_{L^{2 \sigma+2}}^{2 \sigma+2} d s \\
& \leq C\left(C_{0}\right)+C \omega \int_{t_{0}}^{t} \frac{\sinh 2 \omega s}{(\cosh \omega s)^{n \sigma}}\left\|J_{-}^{\omega}(s) u_{-}^{\omega}\right\|_{L^{2}}^{n \sigma} d s .
\end{aligned}
$$

As noticed before, the constants in the last estimate do not depend on $\omega$. Define

$$
y(t):=\sup _{t_{0} \leq s \leq t}\|J(s) u\|_{L^{2}}^{2} .
$$

We have

$$
E_{1}(t) \leq C\left(C_{0}\right)+C y(t)^{n \sigma / 2} \int_{t_{0}}^{t} \frac{\sinh 2 \omega s}{(\cosh \omega s)^{n \sigma}} d s \leq C\left(C_{0}\right)+\frac{C}{\left(\cosh \omega t_{0}\right)^{n \sigma-2}} y(t)^{n \sigma / 2} .
$$

We finally have

$$
y(t) \leq C\left(C_{0}\right)+\frac{C}{\left(\cosh \omega t_{0}\right)^{n \sigma-2}} y(t)^{n \sigma / 2} .
$$

If $\sigma>2 / n$, we conclude by a bootstrap argument, since the constants and $t_{0}$ do not depend on $\omega>0$, and

$$
\cosh \omega t_{0} \underset{\omega \rightarrow+\infty}{\longrightarrow}+\infty \text {. }
$$

This yields a uniform bound for $\|J(t) u\|_{L^{2}}$. The last point of Theorem 1.2 stems from Corollary 3.1.

When $\sigma=2 / n$, the approach is different, as shown in the last section.

\section{The $L^{2}-$ critical case}

Theorem 1.3 is the consequence of two explicit changes of variables. In [5], we noticed that if $u_{+}^{\omega}$ is defined by

$$
u_{+}^{\omega}(t, x)=\frac{1}{(\cos \omega t)^{n / 2}} e^{-i \frac{\omega}{2}|x|^{2} \tan \omega t} v\left(\frac{\tan \omega t}{\omega}, \frac{x}{\cos \omega t}\right),
$$


then $u_{+}^{\omega}$ solves (1.7) with the plus sign and $\sigma=2 / n$. We also proved that $v$ blows up at time $T>0$ if and only if $u_{+}^{\omega}$ blows up at time $\arctan (\omega T) / \omega$. This yields the first point of Theorem 1.3.

Replacing $\omega$ by $\pm i \omega$, define

$$
u_{-}^{\omega}(t, x)=\frac{1}{(\cosh \omega t)^{n / 2}} e^{i \frac{\omega}{2}|x|^{2} \tanh \omega t} v\left(\frac{\tanh \omega t}{\omega}, \frac{x}{\cosh \omega t}\right) .
$$

Then $u_{-}^{\omega}$ solves (1.7) with the minus sign and $\sigma=2 / n$. Now assume that $\lambda<0$, and that $v$ blows up at some finite time $T>0$.

It is easy to see that

$$
\left\|J_{-}^{\omega}(t) u_{-}^{\omega}\right\|_{L^{2}}=\left\|\nabla_{x} v\left(\frac{\tanh \omega t}{\omega}\right)\right\|_{L^{2}} .
$$

Since $\tanh \left(\mathbb{R}_{+}\right)=[0,1[$, if $\omega \geq 1 / T$, then the function of the right hand side of (4.2) does not "see" the time $T$, and $u_{-}^{\omega}$ does not blow up in finite time.

If $\omega<1 / T$, then $u_{-}^{\omega}$ blows up at time

$$
T_{\omega}=\frac{\arg \tanh \omega T}{\omega},
$$

which completes the proof of Theorem 1.3.

More precise results on the influence of $\omega$ on $u_{-}^{\omega}$ are proved in [7].

\section{References}

[1] J. Bourgain and W. Wang, onstruction of blowup solutions for the nonlinear Schrödinger equation with critical nonlinearity, Ann. Scuola Norm. Sup. Pisa Cl. Sci. (4) 25 (1997), no. 1-2, 197-215 (1998).

[2] C. C. Bradley, C. A. Sackett, and R. G. Hulet, Bose-Einstein condensation of Lithium: Observation of limited condensate number, Phys. Rev. Lett. $\mathbf{7 8}$ (1997), 985-989.

[3] C. C. Bradley, C. A. Sackett, J. J. Tollett, and R. G. Hulet, Evidence of BoseEinstein condensation in an atomic gas with attractive interactions, Phys. Rev. Lett. 75 (1995), 1687-1690.

[4] R. Carles, Remarks on nonlinear Schrödinger equations with harmonic potential, Ann. H. Poincaré 3 (2002), no. 4, 757-772.

[5] R. Carles, Critical nonlinear Schrödinger equations with and without harmonic potential, Math. Mod. Meth. Appl. Sci. (M3AS) 12 (2002), no. 10, 1513-1523.

[6] R. Carles, Semi-classical Schrödinger equations with harmonic potential and nonlinear perturbation, Ann. Inst. H. Poincaré Anal. Non Linéaire, 20 (2003), no. $3,501-542$. 
[7] R. Carles, Nonlinear Schrödinger equations with repulsive harmonic potential and applications, SIAM J. Math. Anal., to appear.

[8] R. Carles, C. Fermanian, and I. Gallagher, On the role of quadratic oscillations in nonlinear Schrödinger equations, J. Funct. Anal., to appear.

[9] R. Carles and Y. Nakamura, Nonlinear Schrödinger equations with Stark potential, Hokkaido Math. J., to appear.

[10] T. Cazenave, An introduction to nonlinear Schrödinger equations, Text. Met. Mat., vol. 26, Univ. Fed. Rio de Jan., 1993.

[11] H. L. Cycon, R. G. Froese, W. Kirsch, and B. Simon, Schrödinger operators with application to quantum mechanics and global geometry, study ed., Texts and Monographs in Physics, Springer-Verlag, Berlin, 1987.

[12] T. Cazenave and F. Weissler, Rapidly decaying solutions of the nonlinear Schrödinger equation, Comm. Math. Phys. 147 (1992), 75-100.

[13] A. de Bouard and A. Debussche, Explosion en temps fini pour l'équation de Schrödinger non linéaire stochastique, Séminaire X-EDP, 2002-2003, École Polytech., Exp. No. VII.

[14] A. de Bouard, A. Debussche and L. Di Menza, Theoretical and numerical aspects of stochastic nonlinear Schrödinger equations, Journées Équations aux Dérivées Partielles, Plestin-les-Grèves, 2001, Exp. No. III.

[15] R. P. Feynman and A.R. Hibbs, Quantum mechanics and path integrals (International Series in Pure and Applied Physics), Maidenhead, Berksh.: McGrawHill Publishing Company, Ltd., 365 p., 1965.

[16] R. T. Glassey, On the blowing up of solutions to the Cauchy problem for nonlinear Schrödinger equations, J. Math. Phys. 18 (1977), 1794-1797.

[17] E. B. Kolomeisky, T. J. Newman, J. P. Straley, and X. Qi, Low-dimensional Bose liquids: Beyond the Gross-Pitaevskii approximation, Phys. Rev. Lett. 85 (2000), no. 6, 1146-1149.

[18] F. Merle, Blow-up phenomena for critical nonlinear Schrödinger and Zakharov equations, Proceedings of the International Congress of Mathematicians, Vol. III (Berlin, 1998), no. Extra Vol. III, 1998, pp. 57-66.

[19] F. Merle and P. Raphaël, Blow up Dynamic and Upper Bound on the Blow up Rate fir critical nonlinear Schrödinger Equation, Journées Équations aux Dérivées Partielles, Forges-les-Eaux, 2002, Exp. No. XII.

[20] F. Merle and P. Raphaël, Sharp upper bound on blow up rate for critical non linear Schrödinger equation, Geom. Funct. Anal., to appear.

[21] G. Perelman, On the formation of singularities in solutions of the critical nonlinear Schrödinger equation, Ann. H. Poincaré 2 (2001), no. 4, 605-673. 
[22] W. Thirring, A course in mathematical physics. Vol. 3, Springer-Verlag, New York, 1981, Quantum mechanics of atoms and molecules, Translated from the German by Evans M. Harrell, Lecture Notes in Physics, 141.

[23] T. Tsurumi, H. Morise, and M. Wadati, Stability of Bose-Einstein condensates confined in traps, Internat. J. Modern Phys. B 14 (2000), no. 7, 655-719.

MAB, UMR 5466 CNRS,

UNIVERSITÉ BORDEAUX 1,

351 Cours DE LA LibÉRATiON,

33405 Talence Cedex, France

carles@math.u-bordeaux.fr

www.math.u-bordeaux.fr $/$ carles 